\title{
Counting realizations of Laman graphs on the sphere
}

\author{
Matteo Gallet* \\ International School for Advanced Studies (ISAS/SISSA) \\ Trieste, Italy \\ mgallet@sissa.it \\ Georg Grasegger ${ }^{\dagger}$
}

Johann Radon Institute for Computation and Applied Mathematics (RICAM)

Austrian Academy of Sciences

Linz, Austria

georg.grasegger@ricam. oeaw.ac.at

Josef Schicho $\ddagger$

Research Institute for Symbolic Computation (RISC)

Johannes Kepler University

Linz, Austria

jschicho@risc.jku.at

Submitted: Mar 4, 2019; Accepted: Feb 28, 2020; Published: Apr 3, 2020

(C) The authors. Released under the CC BY-ND license (International 4.0).

\begin{abstract}
We present an algorithm that computes the number of realizations of a Laman graph on a sphere for a general choice of the angles between the vertices. The algorithm is based on the interpretation of such a realization as a point in the moduli space of stable curves of genus zero with marked points, and on the explicit description, due to Keel, of the Chow ring of this space.
\end{abstract}

Mathematics Subject Classifications: 05C99, 51F99, 52C25

\section{Introduction}

Maybe the most important open problem in rigidity theory is the characterization and study of rigid structures in three dimensional space. On the other side, planar structures

*Supported by the Austrian Science Fund (FWF): Erwin Schrödinger Fellowship J4253.

${ }^{\dagger}$ Supported by the Austrian Science Fund (FWF): P31888 and W1214-N15, Project DK9.

${ }^{\ddagger}$ Supported by the Austrian Science Fund (FWF): P31061. 
are reasonably well-understood, in the sense that, for example, we have a characterization of graphs that are generically minimally rigid in the plane. These are the graphs that, once a general assignment for the edge lengths is prescribed, admit only finitely many ways of realizing them in the plane respecting the assignment, if we consider equivalent realizations that differ by an isometry. Pollaczek-Geiringer [Pol27] and Laman [Lam70] described these graphs in terms of their combinatorics, and so they also go under the name of Laman graphs. In $\left[\mathrm{CGG}^{+} 18\right]$, using ideas from tropical geometry, the authors provide a recursive algorithm that computes the number of realizations of a Laman graph for a general assignment of edge lengths, up to plane isometries, if one allows complex coordinates for the points of the realization. It has been proven (see [Pog73, SW07, Izm09, EJN $\left.{ }^{+} 19\right]$ ) that Laman graphs are generically minimally rigid also when we consider realizations on the sphere, so as before one can ask in how many different ways one can realize a Laman graph on the sphere. In this paper, we provide a recursive algorithm that computes this number (again, under the assumption that complex coordinates for the points are allowed) based on a completely different technique from the one used in the planar case. We hope that, although we still work on a surface, moving from the plane to the sphere could be a first step towards determining the number of realizations for generically minimally rigid graphs in three dimensions. For a related work on this topic, discussing real realizations of graphs on the sphere (in addition to the plane and the space), see the recent paper by Bartzos et al. [BELT19]. Among other things, the latter paper proves that for some graphs one can achieve all possible complex realizations via real instances.
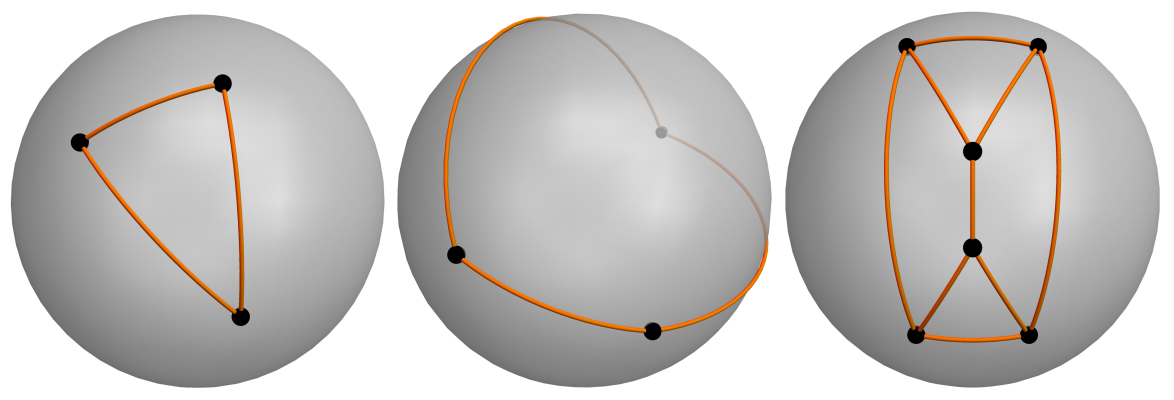

Figure 1: Realizations of graphs on the sphere.

Our result. The main result of this paper is an algorithm that computes the number of realizations of a Laman graph on the (complex) sphere, up to the action of the group $\mathrm{SO}_{3}(\mathbb{C})$ (recall that the real analogue of this group, $\mathrm{SO}_{3}(\mathbb{R})$, is the group of isometries of the real sphere). The key idea is to interpret realizations on the sphere up to $\mathrm{SO}_{3}(\mathbb{C})$ as elements of a moduli space, the so-called moduli space of rational curves with marked points, where each point of the realization corresponds to two marked points. In this interpretation, assigning the distance between two points on the sphere corresponds to prescribing the cross-ratio of the 4 related marked points. By using the properties of the moduli space, in particular the description of its Chow ring and the geometry of some of its divisors, we compute the cardinality of those elements for which the cross-ratios of 
the points corresponding to the edges of a Laman graph are assigned; this provides the answer to our original problem. Remarkably, we have been informed by Gaiane Panina that the moduli space of rational curves with marked points appears also in investigations of flexible polygons, see [NP19].

Structure of the paper. Section 2 describes the problem we want to address in this paper. Section 3 provides the translation from realizations of a graph on the sphere to points of a moduli space of points on the projective line. Section 4 describes, following [Kee92], the moduli space of points on the projective line, and its compactification as moduli space of stable curves of genus zero with marked points; in particular, we recall the description by Keel of the Chow rings of the latter, which plays a key role in the algorithm. Section 5 describes the algorithm. Section 6 reports some computational data obtained using the algorithm.

\section{Realizations of graphs}

A Laman graph is a graph $G=(V, E)$ such that $|E|=2|V|-3$ and $\left|E^{\prime}\right| \leqslant 2\left|V^{\prime}\right|-3$ for every subgraph $G^{\prime}=\left(V^{\prime}, E^{\prime}\right)$. Geiringer [Pol27] and Laman [Lam70] proved that these graphs are generically infinitesimally rigid in the plane. This means the following. A realization of $G$ is a tuple $\left(P_{v}\right)_{v \in V}$ of points in the plane indexed by the vertices of $G$. By applying a Euclidean isometry, we can always suppose that one of the points is the origin $O$ of the plane, and another one lies on the $x$-axis. The set of all possible realizations satisfying the previous two requirements is then given by $\{O\} \times \mathbb{A}_{\mathbb{R}}^{1} \times\left(\mathbb{A}_{\mathbb{R}}^{2}\right)^{n-2}$, where $n$ is the number of vertices. We can now consider the function that computes, for every edge $\{a, b\} \in E$, the distance $d_{\mathbb{A}_{\mathbb{R}}^{2}}\left(P_{a}, P_{b}\right)$ of the corresponding points in a realization. In this way we get a map:

$$
\begin{aligned}
& \Psi_{\mathbb{A}_{\mathbb{R}}^{2}}:\{O\} \times \mathbb{A}_{\mathbb{R}}^{1} \times\left(\mathbb{A}_{\mathbb{R}}^{2}\right)^{n-2} \quad \longrightarrow \quad \mathbb{R}^{|E|} \\
& \left(P_{v}\right)_{v \in V} \quad \mapsto\left(d_{\mathbb{A}_{\mathbb{R}}^{2}}\left(P_{a}, P_{b}\right)^{2}\right)_{\{a, b\} \in E}
\end{aligned}
$$

Notice that the map $\Psi_{\mathbb{A}_{\mathbb{R}}^{2}}$ is a smooth map (better, an algebraic one) between smooth manifolds (better, algebraic varieties) of the same dimension. Laman proved that, if we pick a general point $\vec{P}$ of the domain (namely, if we remove a finite number of "bad" subvarieties from the domain), then the Jacobian of $\Psi_{\mathbb{A}_{\mathbb{R}}^{2}}$ at $\vec{P}$ is invertible, i.e., the map $\Psi_{\mathbb{A}_{\mathbb{R}}^{2}}$ is an isomorphism locally around $P$. Notice that the fibers of $\Psi_{\mathbb{A}_{\mathbb{R}}^{2}}$, namely the sets $\Psi_{\mathbb{A}_{\mathbb{R}}^{2}}^{-1}(\lambda)$ for some $\lambda \in \mathbb{R}^{|E|}$, are the sets of realizations of $G$ where the distances between points, whose corresponding vertices are connected by an edge, are prescribed by $\lambda$. We give a name to these sets:

Definition 1. Let $G=(V, E)$ be a Laman graph and let $\lambda: E \longrightarrow \mathbb{R}$. A realization of $G$ compatible with $\lambda$ is a function $\rho: V \longrightarrow \mathbb{A}_{\mathbb{R}}^{2}$ such that

$$
d_{\mathbb{A}_{\mathbb{R}}^{2}}(\rho(a), \rho(b))^{2}=\lambda(\{a, b\}) \text { for every }\{a, b\} \in E .
$$


When a fiber of $\Psi_{\mathbb{A}_{\mathbb{R}}^{2}}$ is finite - namely when the number of realizations of $G$ compatible with given edge lengths is finite - one may be interested in counting its cardinality, namely the number of ways of realizing a Laman graph with a specified assignment of edge lengths; however, over the real numbers, the cardinality of such fibers may depend on the point in the codomain. Since the result of Laman proves also that the map $\Psi_{\mathbb{A}_{\mathrm{R}}^{2}}$ is dominant, if we pass to the complex numbers we have that, for a general element $\lambda$ in the codomain, the fiber $\Psi_{\mathbb{A}_{\mathbb{C}}^{2}}^{-1}(\lambda)$ is finite and its cardinality does not depend on the point (see [Bum98, Exercise 9.14] $]^{1}$ ). In recent years, there has been some interest in providing lower and upper bounds for this number (see [BS04, ST10, ETV13, GKT18, JO19, BELT19]), and in $\left[\mathrm{CGG}^{+} 18\right]$ the authors provide an iterative formula to compute it, based on tropical geometry. A fully tropical proof of the Geiringer-Laman theorem has recently been provided in [BK19].

One can consider the notion of being generically infinitesimally rigid also on the sphere. It is known that, also on the sphere, the class of generically infinitesimally rigid graphs coincides with the class of Laman graphs (see [Pog73, SW07, Izm09, EJN $\left.{ }^{+} 19\right]$ ). On the sphere, the distance between two points can be taken to be the angle they form (viewed from the origin of the sphere). In this paper we adopt a slightly different definition, which involves the cosine of that angle, because it fits better in the algebraic framework we are going to use. Adopting this definition has no impact as the matter of computing the number of realizations of a graph is concerned. The advantage of this choice is that it provides an algebraic function, which hence allows extensions of fields (in particular, from the real to the complex numbers).

Definition 2. Given two points $P, Q \in S^{2}$, we define their spherical distance as

$$
d_{S^{2}}(P, Q):=\frac{1-\langle P, Q\rangle}{2}
$$

where $\langle P, Q\rangle=\sum_{i=1}^{3} P_{i} Q_{i}$. In particular, if $P$ and $Q$ are antipodal, their spherical distance is 1 .

In this context, we can repeat the same considerations as before: given a configuration $\vec{P}$ on the sphere of a Laman graph $G=(V, E)$, we can always suppose, by applying rotations, that one of the points is $(1,0,0)$ and another lies on a great circle through $(1,0,0)$. We then pass to the complex setting and have that, as in the plane, if $G$ is a Laman graph, then the map

$$
\begin{aligned}
& \Psi_{S_{\mathbb{C}}^{2}}:\{(1,0,0)\} \times S_{\mathbb{C}}^{1} \times\left(S_{\mathbb{C}}^{2}\right)^{n-2} \quad \longrightarrow \quad \mathbb{C}^{|E|} \\
& \left(P_{v}\right)_{v \in V} \quad \mapsto\left(d_{S^{2}}\left(P_{a}, P_{b}\right)\right)_{\{a, b\} \in E}
\end{aligned}
$$

is dominant and its fibers over general points are finite and of constant cardinality. Here, we denoted by $S_{\mathbb{C}}^{2}$ the set $\left\{(x, y, z) \in \mathbb{C}^{3}: x^{2}+y^{2}+z^{2}=1\right\}$, namely the complexification

\footnotetext{
${ }^{1}$ See also the discussion at https://math.stackexchange.com/questions/341281/ number-of-points-in-the-fibre-and-the-degree-of-field-extension
} 
of the sphere, and similarly for the circle $S_{\mathbb{C}}^{1}$. As we remarked, since the function $d_{S^{2}}$ describing the spherical distance is algebraic, we can apply it also to pairs of complex points in $S_{\mathbb{C}}^{2}$. Notice that in the real setting we consider realizations of the graph up to rotations, namely elements of $\mathrm{SO}_{3}(\mathbb{R})$; when we pass to the complex numbers, we consider realizations up to $\mathrm{SO}_{3}(\mathbb{C})$, where

$$
\mathrm{SO}_{3}(\mathbb{C}):=\left\{R \in \mathbb{C}^{3 \times 3}: R R^{t}=R^{t} R=\mathrm{id}, \operatorname{det}(R)=1\right\} .
$$

In this paper, the elements of $\mathrm{SO}_{3}(\mathbb{C})$ will also be (improperly) referred as rotations, or isometries of $S_{\mathbb{C}}^{2}$.

This is the main goal of our paper:

Goal. Compute the cardinality of a general fiber of the map $\Psi_{S_{\mathbb{C}}^{2}}$. In other words, compute the number of realizations of a Laman graph on the sphere compatible with a general assignment of spherical distances for its edges, up to $\mathrm{SO}_{3}(\mathbb{C})$.

We are going to achieve this goal by interpreting realizations up to $\mathrm{SO}_{3}(\mathbb{C})$ as collections of points on the projective complex line $\mathbb{P}_{\mathbb{C}}^{1}$, up to the action of $\mathbb{P G L}_{2}(\mathbb{C})$, namely the group of automorphisms of $\mathbb{P}_{\mathbb{C}}^{1}$. These objects can be interpreted as points in a moduli space, and the explicit description of the intersection theory on that moduli space provides the answer to our question.

\section{Realizations on the sphere as points on a moduli space}

The aim of this section is to show how we can interpret a realization of a graph on the sphere, up to sphere isometries, as a point of the moduli space of stable curves of genus zero with marked points. This provides the theoretical background on which the algorithm presented in Section 5 is based.

We would like to express the spherical distance between two points in $S_{\mathbb{C}}^{2}$ as the crossratio of four points in $\mathbb{P}_{\mathbb{C}}^{1}$. To do so, we associate to each point in $S_{\mathbb{C}}^{2}$ two points in $\mathbb{P}_{\mathbb{C}}^{1}$ via the following construction.

Definition 3. Let

$$
\mathbb{S}_{\mathbb{C}}^{2}=\left\{(x: y: z: w) \in \mathbb{P}_{\mathbb{C}}^{3}: x^{2}+y^{2}+z^{2}-w^{2}=0\right\}
$$

be the projective closure of $S_{\mathbb{C}}^{2}$ in $\mathbb{P}_{\mathbb{C}}^{3}$. Let $A$ be the intersection of $\mathbb{S}_{\mathbb{C}}^{2}$ with the plane at infinity $\{w=0\}$. The conic $A$ is called the absolute conic. Since $\mathbb{S}_{\mathbb{C}}^{2}$ is a smooth quadric in $\mathbb{P}_{\mathbb{C}}^{3}$ there are exactly two families of lines on $\mathbb{S}_{\mathbb{C}}^{2}$; we denote them by $\mathscr{F}_{1}$ and $\mathscr{F}_{2}$. Every point $P \in \mathbb{S}_{\mathbb{C}}^{2}$ is contained in exactly one line $L_{1}$ of $\mathscr{F}_{1}$ and exactly one line $L_{2}$ of $\mathscr{F}_{2}$. The union of these two lines can be obtained by intersecting $\mathbb{S}_{\mathbb{C}}^{2}$ with the tangent plane of $\mathbb{S}_{\mathbb{C}}^{2}$ at $P$. We define the left and the right lifts of $P$ as the intersections of $L_{1}$ and $L_{2}$ with $A$, respectively. We denote them by $P^{l}$ and $P^{r}$, respectively.

Remark 4. Notice that the absolute conic $A$ is a rational curve. This means that, given four points on $A$ (for example, the left and right lifts of two points in $S_{\mathbb{C}}^{2}$ ), we can speak about their cross-ratio. 
Lemma 5. Let $P, Q \in S_{\mathbb{C}}^{2}$. Let $P^{l}, P^{r}$ be the left and right lifts of $P$, and $Q^{l}, Q^{r}$ be the left and right lifts of $Q$. Then

$$
d_{S^{2}}(P, Q)=\frac{\operatorname{cr}\left(P^{l}, P^{r}, Q^{l}, Q^{r}\right)}{\operatorname{cr}\left(P^{l}, P^{r}, Q^{l}, Q^{r}\right)-1}=\operatorname{cr}\left(P^{l}, Q^{r}, Q^{l}, P^{r}\right),
$$

where cr stands for cross-ratio.

Proof. Recall that isometries of $S_{\mathbb{C}}^{2}$ are projective automorphisms of $\mathbb{P}_{\mathbb{C}}^{3}$ leaving the absolute conic $A$ invariant. Hence applying an isometry to $P$ and $Q$ corresponds to applying an automorphism of $\mathbb{P}_{\mathbb{C}}^{1}$ to their lifts, so the cross-ratio of the latter does not change. Hence we can suppose that $P=(1,0,0)$ and $Q=(c, s, 0)$, where $c^{2}+s^{2}=1$. With this choice, we have

$$
d_{S^{2}}(P, Q)=\frac{1-c}{2} .
$$

A direct computation shows that, since the tangent planes at $P$ and $Q$ have equations $x-w=0$ and $c x+s y-w=0$, respectively:

$$
\begin{array}{ll}
P^{l}=(0: i: 1: 0), & P^{r}=(0:-i: 1: 0), \\
Q^{l}=(-i s: i c: 1: 0), & Q^{r}=(i s:-i c: 1: 0) .
\end{array}
$$

In order to compute the cross-ratio, we take an isomorphism between $A$ and $\mathbb{P}_{\mathbb{C}}^{1}$, for example the one given by the projection of $A$ from the point $(i: 0: 1: 0)$ to the line $\{z=w=0\}$. The projections of the previous four points are

$$
(-1: 1: 0: 0),(1: 1: 0: 0),(-1-s: c: 0: 0),(1-s: c: 0: 0) \text {. }
$$

Their cross-ratio is

$$
\left(\frac{-1-s}{c}+1\right)\left(\frac{1-s}{c}-1\right) /\left(\left(\frac{-1-s}{c}-1\right)\left(\frac{1-s}{c}+1\right)\right) .
$$

A direct computation then proves the statement.

Proposition 6. Let $\vec{P}=\left(P_{1}, \ldots, P_{n}\right)$ and $\vec{Q}=\left(Q_{1}, \ldots, Q_{n}\right)$ be two $n$-tuples of points in $S_{\mathbb{C}}^{2}$. Denote by $P_{i}^{l}, P_{i}^{r}$ and $Q_{i}^{l}, Q_{i}^{r}$ the left and right lifts of $P_{i}$ and $Q_{i}$, respectively, for all $i \in\{1, \ldots, n\}$. Then $\vec{P}$ and $\vec{Q}$ differ by an isometry of $S_{\mathbb{C}}^{2}$ if and only if $\left(\overrightarrow{P^{l}}, \overrightarrow{P^{r}}\right)$ and $\left(\overrightarrow{Q^{l}}, \overrightarrow{Q^{r}}\right)$ differ by an element of $\mathbb{P G L}_{2}(\mathbb{C})$.

Proof. Every isometry of $S_{\mathbb{C}}^{2}$ is a projective automorphism of $\mathbb{P}_{\mathbb{C}}^{3}$ leaving the absolute conic $A$ invariant. This means that every isometry of $S_{\mathbb{C}}^{2}$ determines an automorphism of $A$. In this way we get a map

$$
\mathrm{SO}_{3}(\mathbb{C}) \longrightarrow \mathbb{P G L}(\mathbb{C}),
$$

which is a homomorphism of Lie groups. Our statement is proven if we can show that this is an isomorphism. Suppose that we have an isometry of $S_{\mathbb{C}}^{2}$ that induces the identity on $A$. 
Then the corresponding projective automorphism of $\mathbb{P}_{\mathbb{C}}^{3}$ fixes the whole plane at infinity, and also the center of $S_{\mathbb{C}}^{2}$ : the only element in $\mathrm{SO}_{3}(\mathbb{C})$ satisfying these requirements is the identity. Hence, the homomorphism is injective. Since the two Lie groups have the same dimension and are both connected, then the homomorphism is also surjective. This concludes the proof.

Remark 7 . Notice that, in general, it is not true that $n$ distinct points on $S_{\mathbb{C}}^{2}$ determine $2 n$-tuples of distinct points on the absolute conic via the lift operation. In fact, this fails precisely when two points on $S_{\mathbb{C}}^{2}$ belong to the same line. If $P, Q \in S_{\mathbb{C}}^{2}$ with $P=(\alpha, \beta, \gamma)$ and $Q=\left(\alpha^{\prime}, \beta^{\prime}, \gamma^{\prime}\right)$, then $P$ and $Q$ belong to the same line when, considered as points in $\mathbb{P}_{\mathbb{C}}^{3}$, they are orthogonal with respect to the quadratic form determined by the equation of $\mathbb{S}_{\mathbb{C}}^{2}$, namely if:

$$
\left(\begin{array}{llll}
\alpha & \beta & \gamma & 1
\end{array}\right)\left(\begin{array}{cccc}
1 & & & \\
& 1 & & \\
& & 1 & \\
& & & -1
\end{array}\right)\left(\begin{array}{l}
\alpha^{\prime} \\
\beta^{\prime} \\
\gamma^{\prime} \\
1
\end{array}\right)=0 \Leftrightarrow\left\langle\left(\begin{array}{l}
\alpha \\
\beta \\
\gamma
\end{array}\right),\left(\begin{array}{l}
\alpha^{\prime} \\
\beta^{\prime} \\
\gamma^{\prime}
\end{array}\right)\right\rangle-1=0
$$

Hence, if we suppose that $\vec{P}$ is a realization of a Laman graph $G$ compatible with a general assignment of spherical distances for its edges, then $\vec{P}$ determines a $2 n$-tuple of distinct points $\left(\overrightarrow{P^{l}}, \overrightarrow{P^{r}}\right)$ on the absolute conic $A$.

The combination of Lemma 5, Proposition 6, and Remark 7 shows that, instead of considering $n$-tuples $\vec{P}$ that are realizations of a Laman graph compatible with a general assignment of spherical distances for its edges, up to the action of $\mathrm{SO}_{3}(\mathbb{C})$, we can consider $2 n$-tuples $\left(\overrightarrow{P^{l}}, \overrightarrow{P^{r}}\right)$ of points on $\mathbb{P}_{\mathbb{C}}^{1}$ for which some cross-ratios are assigned, up to the action of $\mathbb{P G L}_{2}(\mathbb{C})$. The latter are elements of a so-called moduli space of curves of genus zero with marked points. In the next section we describe this object and its properties concerning intersection theory. Afterwards, we come back to our original problem and cast it into this theoretical framework.

\section{The moduli space of stable curves of genus zero with marked points}

In this section we describe for the reader's convenience the well-known moduli space of stable curves of genus zero with marked points and its Chow ring, following [Kee92]. No new results are presented in this section.

Let us start by recalling a basic and fundamental result in projective geometry: every triple of distinct points $P, Q$, and $R$ in $\mathbb{P}_{\mathbb{C}}^{1}$ can be mapped to the triple $(1: 0),(0: 1)$, and $(1: 1)$ via a unique automorphism of $\mathbb{P}_{\mathbb{C}}^{1}$, namely an element of $\mathbb{P G L}_{2}(\mathbb{C})$. Hence, any triple of distinct points in $\mathbb{P}_{\mathbb{C}}^{1}$ is projectively equivalent to any other one, namely there always exists an automorphism mapping one to the other. If we consider a 4-tuple of distinct points $P, Q, R, S$, then there is a unique element that takes it to the 4-tuple $(1: 0),(0: 1),(1: 1)$, and $(1: \lambda)$ : the number $\lambda$ is called the cross-ratio of the tuple 
$(P, Q, R, S)$. Two 4-tuples of distinct points are then projectively equivalent if and only if their cross-ratios are the same. Hence, each equivalence class of 4 -tuples of distinct points modulo $\mathbb{P G L}_{2}(\mathbb{C})$ is represented by its cross-ratio, namely by an element in $\mathbb{C} \backslash\{0,1\}$, or equivalently in $\mathbb{P}_{\mathbb{C}}^{1} \backslash\{(1: 0),(0: 1),(1: 1)\}$. We then say that $\mathbb{P}_{\mathbb{C}}^{1} \backslash\{(1: 0),(0: 1),(1: 1)\}$ is the moduli space of 4 -tuples of distinct points in $\mathbb{P}_{\mathbb{C}}^{1}$.

We can consider, for every $m \geqslant 4$, the space of equivalence classes of $m$-tuples of distinct points under the action of $\mathbb{P G L}_{2}(\mathbb{C})$ : each such equivalence class is uniquely determined by an element in the quasi-projective variety

$$
\left(\mathbb{P}_{\mathbb{C}}^{1} \backslash\{(1: 0),(0: 1),(1: 1)\}\right)^{m-3} \backslash\left\{\begin{array}{c}
(m-3) \text {-tuples having at least } \\
\text { two equal components }
\end{array}\right\}
$$

This space is called the moduli space of $m$-tuples of distinct points in $\mathbb{P}_{\mathbb{C}}^{1}$, and is denoted by $\mathscr{M}_{0, m}$. One may notice that this space is not compact under the Euclidean topology (in more algebro-geometric terms, it is not complete), and this may be a problem when dealing with enumerative questions, as the one we address in this work. Because of this, researchers focused on finding compactifications of these moduli spaces. A possible smooth compactification of the space $\mathscr{M}_{0, m}$, denoted $\overline{\mathscr{M}}_{0, m}$, was constructed by Knudsen [Knu83] (see [Mum65, Mum77, Gie82] for a more general account on the topic). This construction introduces a boundary for $\mathscr{M}_{0, m}$, constituted of particular curves, called stable curves, which are essentially reducible curves whose irreducible components are rational curves, intersecting in nodes. More precisely, we have:

Definition 8 ([Kee92, Introduction]). A stable curve of genus zero with $m$ marked points is a reduced, possibly reducible, curve $C$ with at worst node singularities, together with $m$ distinct marked points $p_{1}, \ldots, p_{m}$ on it such that:

- the points $\left\{p_{i}\right\}_{i=1}^{m}$ lie on the smooth locus of $C$;

- each irreducible component of $C$ is isomorphic to $\mathbb{P}_{\mathbb{C}}^{1}$, and altogether all irreducible components form a tree;

- for each irreducible component of $C$, the sum of the numbers of singular points and of marked points on that component is at least 3 .

The geometry of $\overline{\mathscr{M}}_{0, m}$ is rich and well-studied: we refer to [Kee92, Introduction], [Kap93], and [KV07, Chapter 1] for a discussion.

As we are going to see in Section 5, our algorithm relies on the understanding of how subvarieties of $\overline{\mathscr{M}}_{0, m}$ intersect each other. This piece of information is encoded in the so-called Chow ring, which is a standard object in intersection theory. For its definition and properties we refer to the introduction [Ful84], or to the standard book [Ful98].

For the reader's convenience, we briefly provide in Theorem 11 the description by Keel of the Chow ring of $\overline{\mathscr{M}}_{0, m}$. First, we need to introduce some particular divisors that Keel calls "vital". 
Definition 9 ([Kee92, Introduction $])$. Let $(I, J)$ be a partition of $\{1, \ldots, m\}$ where $|I| \geqslant 2$ and $|J| \geqslant 2$. We define the divisor $D_{I, J}$ in $\mathscr{\mathscr { M }}_{0, m}$ to be the divisor whose general point is a stable curve with two irreducible components such that the marked points labeled by $I$ lie on one component, while the marked points labeled by $J$ lie on the other component.

Proposition 10 (Knudsen, [Kee92, Introduction and Fact 2]). Any divisor $D_{I, J}$ as in Definition 9 is smooth and it is isomorphic to the product $\overline{\mathscr{M}}_{0,|I|+1} \times \overline{\mathscr{M}}_{0,|J|+1}$; in the previous isomorphism, the point of intersection of the two components of a general stable curve in $D_{I, J}$ counts as an extra marked point in each of the two factors of the product.

Theorem 11 ([Kee92, Introduction and Theorem 1]). The Chow ring of $\overline{\mathscr{M}}_{0, m}$ admits the following description:

$$
\mathbb{Z}\left[D_{I, J}:(I, J) \text { is a partition of }\{1, \ldots, m\} \text { where }|I| \geqslant 2 \text { and }|J| \geqslant 2\right] / \sim,
$$

where the equivalence $\sim$ is given by the relations:

- $D_{I, J}=D_{J, I}$,

- for any four distinct elements $a, b, c, d \in\{1, \ldots, m\}$ we have

$$
\sum_{\substack{a, b \in I \\ c, d \in J}} D_{I, J}=\sum_{\substack{a, c \in I \\ b, d \in J}} D_{I, J}=\sum_{\substack{a, d \in I \\ b, c \in J}} D_{I, J},
$$

- $D_{I, J} \cdot D_{K, L}=0$ unless one of the following holds:

$$
I \subseteq K, \text { or } K \subseteq I, \text { or } J \subseteq L, \text { or } L \subseteq J
$$

Moreover, the three sums in Equation $(\triangle)$ are the pullbacks of the respective divisors $D_{\{a, b\},\{c, d\}}, D_{\{a, c\},\{b, d\}}$, and $D_{\{a, d\},\{b, c\}}$ under the map

$$
\pi_{a, b, c, d}: \overline{\mathscr{M}}_{0, m} \longrightarrow \overline{\mathscr{M}}_{0,4}^{a, b, c, d} \cong \mathbb{P}_{\mathbb{C}}^{1} .
$$

Here, the map $\pi_{a, b, c, d}$ is the map that forgets all the marked points except for the ones labeled by $a, b, c$, and $d$. We put the superscript $(\cdot)^{a, b, c, d}$ on its codomain to denote that its elements are stable curves with marked points labeled by $a, b, c, d$.

\section{The algorithm}

At the end of Section 2 we understood that realizations on the sphere up to $\mathrm{SO}_{3}(\mathbb{C})$ can be considered as elements of a moduli space. In particular, configurations of $n$ points on the complex sphere up to $\mathrm{SO}_{3}(\mathbb{C})$ correspond to configurations of $2 n$ points on a rational curve up to $\mathbb{P G L}_{2}(\mathbb{C})$, i.e., elements in the moduli space of rational curves with marked points. Moreover, assigning angles between two points on a sphere corresponds to assigning the cross-ratio of the 4-tuple constituted of the left and right lifts of those two points. The 
elements of the moduli space, for which the cross-ratio of 4 marked points is prescribed, are fibers of the map

$$
\overline{\mathscr{M}}_{0,2 n} \longrightarrow \overline{\mathscr{M}}_{0,4} \cong \mathbb{P}_{\mathbb{C}}^{1}
$$

that forgets all but the 4 considered marked points (see Theorem 11). Hence, the elements of the moduli space we are interested in, namely the ones for which the cross-ratios are specified for the 4-tuples arising from edges of a graph, are fibers of products of these maps. We give a name to these maps:

Definition 12. Let $G=(V, E)$ be a graph, and suppose that $V=\{1, \ldots, n\}$. We define the morphism

$$
\Phi_{G}: \overline{\mathscr{M}}_{0,2 n} \longrightarrow \prod_{\{a, b\} \in E} \overline{\mathscr{M}}_{0,4}^{a, b, a+n, b+n}
$$

whose components are the maps $\pi_{a, b, a+n, b+n}: \overline{\mathscr{M}}_{0,2 n} \longrightarrow \overline{\mathscr{M}}_{0,4}$ forgetting all but 4 marked points, defined in Theorem 11. The choice for the indices reflects the labeling $\left(P_{1}^{l}, \ldots, P_{n}^{l}\right.$, $\left.P_{1}^{r}, \ldots, P_{n}^{r}\right)$ of the marked points on the rational curves, putting first the points corresponding to left lifts, and then the points corresponding to right lifts of points on the sphere. The interpretation of realizations of a graph in $S_{\mathbb{C}}^{2}$ as points in the moduli space $\overline{\mathscr{M}}_{0,2 n}$ and of spherical distances as cross ratios we provided in Section 2 tells us that when $G$ is a Laman graph, the map $\Phi_{G}$ is a dominant morphism between smooth varieties of the same dimension, so its general fibers are constituted of finitely many points. In fact, the Laman condition implies that

$$
\operatorname{dim} \overline{\mathscr{M}}_{0,2 n}=2 n-3=\operatorname{dim} \prod_{\{a, b\} \in E} \overline{\mathscr{M}}_{0,4}^{a, b, a+n, b+n},
$$

so the domain and the codomain of $\Phi_{G}$ have the same dimension. Moreover, since a Laman graph has (complex) realizations for a general choice of edge lengths, we have that $\Phi_{G}$ is dominant. The fact that its general fibers are constituted of finitely many points follows then from general results about maps between varieties (see [Sha13, Chapter 1, Section 6, Theorem 1.25]).

Remark 13 . For any Laman graph $G$ with $n$ vertices, the image of the boundary of $\overline{\mathscr{M}}_{0,2 n}$ under $\Phi_{G}$ is a proper subvariety of $\prod_{\{a, b\} \in E} \overline{\mathscr{M}}_{0,4}^{a, b, a+n, b+n}$. In fact, since the domain and the codomain of $\Phi_{G}$ have the same dimension, and the boundary has codimension 1 in $\overline{\mathscr{M}}_{0,2 n}$, it follows that its image under $\Phi_{G}$ has codimension at least 1 in the codomain of $\Phi_{G}$. This means that a general fiber of $\Phi_{G}$ will not intersect the boundary, and so it is constituted of classes of rational curves with $2 n$ distinct marked points. Each of such rational curves is then isomorphic to the absolute conic $A$, and the $2 n$ marked points determine a realization of $G$ on the sphere. Moreover, when $G$ is a Laman graph, a general fiber of $\Phi_{G}$ is a complete intersection in $M_{0,2 n}$ and it is constituted by reduced points.

The discussion so far proves the following theorem.

Theorem 14. Given a Laman graph $G$, then the number of realizations of $G$ in $S_{\mathbb{C}}^{2}$ for a general assignment of spherical distances for its edges, up to $\mathrm{SO}_{3}(\mathbb{C})$, equals the cardinality of a general fiber of the map $\Phi_{G}$ as in Definition 12. 
In the light of Theorem 14, our goal becomes, given a Laman graph $G$, to compute the cardinality of a general fiber of $\Phi_{G}$.

Remark 15. Let us describe how the class in the Chow ring of a fiber of $\Phi_{G}$ looks like. Since the codomain of $\Phi_{G}$ is a product, we can express a fiber as the intersection of the fibers of the maps to each component of the product. In other words, if we fix $\Lambda \in \prod_{\{a, b\} \in E} \overline{\mathscr{M}}_{0,4}^{a, b, a+n, b+n}$ and we denote by $\Phi_{a, b}$ the composition of $\Phi_{G}$ with the projection to the factor $\mathscr{\mathscr { M }}_{0,4}^{a, b, a+n, b+n}$, then we have that

$$
\Phi_{G}^{-1}(\Lambda)=\bigcap_{\{a, b\} \in E} \Phi_{a, b}^{-1}\left(\Lambda_{a, b}\right) .
$$

It follows then, using the notation of Theorem 11, that the class of $\Phi_{G}^{-1}(\Lambda)$ in the Chow ring of $\overline{\mathscr{M}}_{0,2 n}$ is given by

$$
\prod_{\{a, b\} \in E} \sum_{\substack{a, b \in I \\ a+n, b+n \in J}} D_{I, J} .
$$

Since a general fiber of $\Phi_{G}$ is a reduced complete intersection in $\overline{\mathscr{M}}_{0,2 n}$ when $G$ is a Laman graph, its cardinality is the degree of its Chow class. Hence we get:

Proposition 16. Given a Laman graph $G=(V, E)$ with $V=\{1, \ldots, n\}$, the number of realizations of $G$ in $S_{\mathbb{C}}^{2}$ for a general assignment of spherical distances for its edges, up to $\mathrm{SO}_{3}(\mathbb{C})$, equals

$$
\operatorname{deg}\left(\prod_{\{a, b\} \in E} \sum_{\substack{a, b \in I \\ a+n, b+n \in J}} D_{I, J}\right) .
$$

Algorithm CountRealizations computes the degree in Proposition 16 using the description of vital divisors $D_{I, J}$ provided by Proposition 10 and the relations in the Chow ring stated in Theorem 11.

In fact, if we fix an edge $\left\{a_{0}, b_{0}\right\} \in E$ of a Laman graph $G=(V, E)$, then the class we want to compute is

$$
\left(\sum_{\substack{a_{0}, b_{0} \in I_{0} \\ a_{0}+n, b_{0}+n \in J_{0}}} D_{I_{0}, J_{0}}\right) \cdot \underbrace{\left(\prod_{\{a, b\} \in E \backslash\left\{a_{0}, b_{0}\right\}} \sum_{\substack{a, b \in I \\ a+n, b+n \in J}} D_{I, J}\right)}_{=: F_{a_{0}, b_{0}}} .
$$

For every $\left(I_{0}, J_{0}\right)$ such that $a_{0}, b_{0} \in I_{0}$ and $a_{0}+n, b_{0}+n \in J_{0}$, the product $D_{I_{0}, J_{0}} \cdot F_{a_{0}, b_{0}}$ can be computed by restricting $F_{a_{0}, b_{0}}$ to $D_{I_{0}, J_{0}}$ and using the isomorphism $D_{I_{0}, J_{0}} \cong \overline{\mathscr{M}}_{0,\left|I_{0}\right|+1} \times$ $\overline{\mathscr{M}}_{0,\left|J_{0}\right|+1}$. We show that both the restrictions of $F_{a_{0}, b_{0}}$ to $\overline{\mathscr{M}}_{0,\left|I_{0}\right|+1}$ and to $\overline{\mathscr{M}}_{0,\left|J_{0}\right|+1}$ have the same structure of the initial class we wanted to compute, and this determines a recursive procedure to solve our task.

To clarify the recursive procedure, let us start by noticing that the class

$$
\prod_{\{a, b\} \in E} \sum_{\substack{a, b \in I \\ a+n, b+n \in J}} D_{I, J}
$$




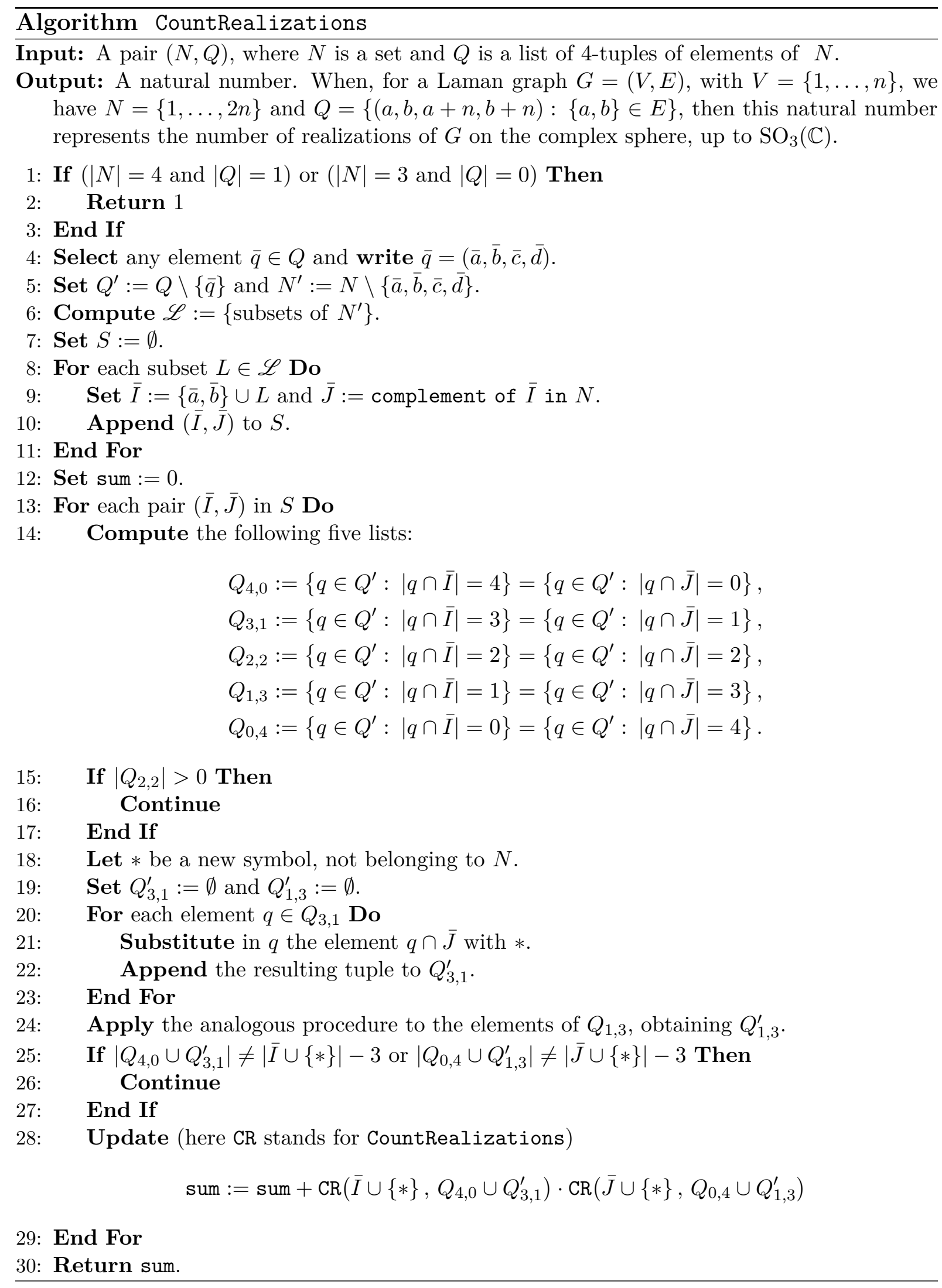


from Proposition 16 is a particular instance of a more general construction. The construction works as follows: we start from a set $N$, and a set $Q$ of 4-tuples of distinct elements of $N$. From now on, we use the notation $\mathscr{M}_{0, N}$ to denote the moduli space of stable rational curves where the marked points are labeled by $N$. Then we form the following class in the Chow ring of $\overline{\mathscr{M}}_{0, N}$ :

$$
\mathcal{A}_{N, Q}:=\prod_{\substack{q \in Q \\ q=(a, b, c, d)}} \sum_{\substack{a, b \in I \\ c, d \in J}} D_{I, J} .
$$

Notice that if we set $N_{0}:=\{1, \ldots, 2 n\}$ and $Q_{0}:=\{(a, b, a+n, b+n):\{a, b\} \in E\}$, then the class from Equation (2) equals $\mathcal{A}_{N_{0}, Q_{0}}$. In this perspective, also the class $F_{a_{0}, b_{0}}$ from Equation (1) can be seen as a particular case of a general construction: what we do here is to select some $\bar{q} \in Q$, with $\bar{q}=(\bar{a}, \bar{b}, \bar{c}, \bar{d})$, and to define the class

$$
\mathcal{G}_{\bar{q}}:=\prod_{\substack{q \in Q \backslash\{\bar{q}\} \\ q=(a, b, c, d)}} \sum_{\substack{a, b \in I \\ c, d \in J}} D_{I, J},
$$

so that we get the factorization

$$
\mathcal{A}_{N, Q}=\left(\sum_{\substack{\bar{a}, \bar{b} \in \bar{I} \\ \bar{c}, \bar{d} \in \bar{J}}} D_{\bar{I}, \bar{J}}\right) \cdot \mathcal{G}_{\bar{q}}
$$

With this notation, the class $F_{a_{0}, b_{0}}$ equals $\mathcal{G}_{q_{0}}$, where $q_{0}=\left(a_{0}, b_{0}, a_{0}+n, b_{0}+n\right)$.

We show now that we can set up an iterative procedure for the computation of the degree of classes of type $\mathcal{A}_{N, Q}$. Notice that, taking into account Equation (4), this can be achieved once we are able to compute the degree of a product $D_{\bar{I}, \bar{J}} \cdot \mathcal{G}_{\bar{q}}$. We then fix $\bar{q} \in Q$ with $\bar{q}=(\bar{a}, \bar{b}, \bar{c}, \bar{d})$ and we select a pair $(\bar{I}, \bar{J})$ such that $\bar{a}, \bar{b} \in \bar{I}$ and $\bar{c}, \bar{d} \in \bar{J}$. If there exists $q \in Q \backslash\{\bar{q}\}$ such that $|q \cap \bar{I}|=|q \cap \bar{J}|=2$, then the restriction of $\mathcal{G}_{\bar{q}}$ to $D_{\bar{I}, \bar{J}}$ is zero by [Kee92, Fact 2]. Otherwise, the restriction of $\mathcal{G}_{\bar{q}}$ to $D_{\bar{I}, \bar{J}} \cong \overline{\mathscr{M}}_{0, \bar{I} \cup\{*\}} \times \overline{\mathscr{M}}_{0, \bar{J} \cup\{*\}}$ is the product of two classes $\mathcal{G}_{\bar{q}}^{\bar{I}}$ and $\mathcal{G}_{\bar{q}}^{\bar{J}}$. Recall, in fact, that the Chow ring of $\overline{\mathscr{M}}_{0, \bar{I} \cup\{*\}} \times \overline{\mathscr{M}}_{0, \bar{J} \cup\{*\}}$ is the tensor product of the Chow rings of $\overline{\mathscr{M}}_{0, \bar{I} \cup\{*\}}$ and $\overline{\mathscr{M}}_{0, \bar{J} \cup\{*\}}$ by [Kee92, Theorem 2]. Analyzing the isomorphism making $D_{\bar{I}, \bar{J}}$ into a product (see [Kee92, Fact 2] and [Knu83, Theorem 3.7]), one sees that the two classes $\mathcal{G}_{\bar{q}}^{\bar{I}}$ and $\mathcal{G}_{\bar{q}}^{\bar{J}}$ admit the following description. For $k \in\{0, \ldots, 4\}$, define the sets:

$$
Q_{k, 4-k}:=\{q \in Q \backslash\{\bar{q}\}:|q \cap \bar{I}|=k\}=\{q \in Q \backslash\{\bar{q}\}:|q \cap \bar{J}|=4-k\} .
$$

Notice that, by definition, all tuples in $Q_{3,1}$ have exactly one element in $\bar{J}$. Define $Q_{3,1}^{\prime}$ to be set obtained by substituting in all 4-tuples of $Q_{3,1}$ their element in $\bar{J}$ by the new symbol *. Analogously, define $Q_{1,3}^{\prime}$. Then the classes $\mathcal{G}_{\bar{q}}^{\bar{I}}$ and $\mathcal{G}_{\bar{q}}^{\bar{J}}$ are

$$
\prod_{\substack{q \in Q_{4,0} \cup Q_{3,1}^{\prime} \\ q=(a, b, c, d)}} \sum_{\substack{a, b \in K \\ c, d \in L}} D_{K, L} \quad \text { and } \prod_{\substack{q \in Q_{0,4} \cup Q_{1,3}^{\prime} \\ q=(a, b, c, d)}} \sum_{\substack{a, b \in K \\ c, d \in L}} D_{K, L},
$$


where the $D_{K, L}$ are divisors in the appropriate moduli spaces, namely for $\mathcal{G}_{\bar{q}}^{\bar{I}}$ they are divisors in $\overline{\mathscr{M}}_{0, \bar{I} \cup\{*\}}$, while for $\mathcal{G}_{\bar{q}}^{\bar{J}}$ they are divisors in $\overline{\mathscr{M}}_{0, \bar{J} \cup\{*\}}$.

Hence, in the notation of Equation (3), if we set

$$
\begin{array}{ll}
N_{\bar{I}}:=\bar{I} \cup\{*\}, & Q_{\bar{I}}:=Q_{4,0} \cup Q_{3,1}^{\prime}, \\
N_{\bar{J}}:=\bar{J} \cup\{*\}, & Q_{\bar{J}}:=Q_{0,4} \cup Q_{1,3}^{\prime},
\end{array}
$$

we have

$$
\mathcal{G}_{\bar{q}}^{\bar{I}}=\mathcal{A}_{N_{\bar{I}}, Q_{\bar{I}}} \quad \text { and } \quad \mathcal{G}_{\bar{q}}^{\bar{J}}=\mathcal{A}_{N_{\bar{J}}, Q_{\bar{J}}} .
$$

Therefore, the degree of a class $\mathcal{A}_{N, Q}$ can be computed by fixing an element $\bar{q} \in Q$, $\bar{q}=(\bar{a}, \bar{b}, \bar{c}, \bar{d})$, and using Equation (4), thus obtaining the formula

$$
\operatorname{deg} \mathcal{A}_{N, Q}=\sum_{\substack{\bar{a}, \bar{b} \in \bar{I} \\ \bar{c}, \bar{d} \in \bar{J}}}\left(\operatorname{deg} \mathcal{A}_{N_{\bar{I}}, Q_{\bar{I}}} \cdot \operatorname{deg} \mathcal{A}_{N_{\bar{J}}, Q_{\bar{J}}}\right)
$$

where it is intended that a summand is zero if the corresponding set $Q_{2,2}$ is not empty. This allows one to set up a recursive procedure for the computation of the degree of a class $\mathcal{A}_{N, Q}$ - so in particular of the class of a fiber of $\Phi_{G}$. The recursion stops if we reach one of these situations:

- The set $Q_{2,2}$ is not empty: in this case we can skip the contribution given by this class, since its degree is zero.

- The set $N$ is composed of four elements, and $Q$ consists of a single tuple: in this case the degree of the class is 1 .

- The cardinality of $Q_{4,0} \cup Q_{3,1}^{\prime}$ is different from $|\bar{I} \cup\{*\}|-3$ or the cardinality of $Q_{0,4} \cup Q_{1,3}^{\prime}$ is different from $|\bar{J} \cup\{*\}|-3$ : in this case either $\mathcal{G}_{\bar{q}}^{\bar{I}}$ or $\mathcal{G}_{\bar{q}}^{\bar{J}}$ is zero, and so this contribution can be skipped.

The discussion so far proves the correctness of Algorithm CountRealizations. Termination is implied by the fact that the size of the sets always decreases and therefore, the base cases are reached.

\section{Computed Data}

Using Algorithm CountRealizations we computed the number of realizations on the sphere of all Laman graphs with up to 10 vertices. Table 1 lists those graphs that have the maximal number of realizations on the sphere within the class of graphs with the same number of vertices.

Remark 17. The paper [BELT19] shows that the number of real spherical realizations matches the number of complex ones for some graphs in Table 1 (all graphs with 6 and 7 vertices, and one of the graphs with 8 vertices). 
Table 1: Graphs with maximal number of complex realizations on the sphere within graphs of $n$ vertices. $\mathrm{Lam}_{2}$ denotes the number of complex realizations in the plane.

(sraph(s)

Note that up till 8 vertices the graph with maximal Laman number (i.e., number of realizations in the plane) is also in the list of graphs with maximal number of realizations on the sphere. However, the graph with maximal Laman number with 9 vertices is different from the one with maximal number of realizations on the sphere. The latter has a very particular structure (see last row of Table 1).

Our recursive algorithm gives a significant improvement over the naive approach, which is to determine the number of solutions via a Gröbner basis computation. Furthermore, the Gröbner basis approach needs randomly fixed edge lengths where CountRealizations computes the numbers symbolically. For a graph with 9 vertices and maximal number of realizations (see Table 1) our algorithm needs 5.66s in Mathematica and 3.57s in Python. The Gröbner basis computation needed 5850s in Mathematica and 27s in Maple. 


\section{Acknowledgments.}

We thank the Erwin Schrödinger Institute (ESI) of the University of Vienna for the hospitality during the workshop "Rigidity and Flexibility of Geometric Structures", when this project started. We thank the Austrian Science Fund (FWF) W1214-N15, Project DK9, for proving financial support for scientific travels and meetings. We thank Anthony Nixon for providing us with useful references concerning rigidity of graphs on the sphere. We thank Jan Legerský for helpful discussions on the topic of this paper.

\section{References}

[BELT19] Evangelos Bartzos, Ioannis Z. Emiris, Jan Legerský, and Elias Tsigaridas, On the maximal number of real embeddings of minimally rigid graphs in $\mathbb{R}^{2}, \mathbb{R}^{3}$ and $S^{2}$, Journal of Symbolic Computation in press (2019), https://doi.org/10.1016/j.jsc.2019.10.015.

[BK19] Daniel I. Bernstein and Robert Krone, The tropical Cayley-Menger variety, SIAM Journal on Discrete Mathematics 33 (2019), no. 3, 1725-1742.

[BS04] Ciprian Borcea and Ileana Streinu, The number of embeddings of minimally rigid graphs, Discrete \& Computational Geometry 31 (2004), 287-303.

[Bum98] Daniel Bump, Algebraic geometry, World Scientific Publishing Co., Inc., River Edge, NJ, 1998.

[CGG $\left.{ }^{+} 18\right]$ Jose Capco, Matteo Gallet, Georg Grasegger, Christoph Koutschan, Niels Lubbes, and Josef Schicho, The number of realizations of a Laman graph, SIAM Journal on Applied Algebra and Geometry 2 (2018), no. 1, 94-125.

$\left[\mathrm{EJN}^{+} 19\right]$ Yaser Eftekhari, Bill Jackson, Anthony Nixon, Bernd Schulze, Shin-ichi Tanigawa, and Walter Whiteley, Point-hyperplane frameworks, slider joints, and rigidity preserving transformations, Journal of Combinatorial Theory, Series B 135 (2019), 48-74.

[ETV13] Ioannis Z. Emiris, Elias P. Tsigaridas, and Antonios Varvitsiotis, Mixed Volume and Distance Geometry Techniques for Counting Euclidean Embeddings of Rigid Graphs, Distance Geometry: Theory, Methods, and Applications, pp. 23-45, Springer New York, New York, NY, 2013.

[Ful84] William Fulton, Introduction to intersection theory in algebraic geometry, CBMS Regional Conference Series in Mathematics, vol. 54, Published for the Conference Board of the Mathematical Sciences, Washington, DC; by the American Mathematical Society, Providence, RI, 1984.

[Ful98] _ Intersection theory, second ed., Ergebnisse der Mathematik und ihrer Grenzgebiete. 3. Folge. A Series of Modern Surveys in Mathematics [Results in Mathematics and Related Areas. 3rd Series. A Series of Modern Surveys in Mathematics], vol. 2, Springer-Verlag, Berlin, 1998. 
[Gie82] David Gieseker, Lectures on moduli of curves, Tata Institute of Fundamental Research Lectures on Mathematics and Physics, vol. 69, Published for the Tata Institute of Fundamental Research, Bombay; Springer-Verlag, BerlinNew York, 1982.

[GKT18] Georg Grasegger, Christoph Koutschan, and Elias Tsigaridas, Lower bounds on the number of realizations of rigid graphs, Experimental Mathematics 0 (2018), no. 0, 1-12, online first.

[Izm09] Ivan Izmestiev, Projective background of the infinitesimal rigidity of frameworks, Geometriae Dedicata 140 (2009), no. 1, 183-203.

[JO19] Bill Jackson and John C. Owen, Equivalent realisations of a rigid graph, Discrete Applied Mathematics 256 (2019), 42-58.

[Kap93] Mikhail M. Kapranov, Veronese curves and Grothendieck-Knudsen moduli space $\bar{M}_{0, n}$, Journal of Algebraic Geometry 2 (1993), no. 2, 239-262.

[Kee92] Sean Keel, Intersection theory of moduli space of stable n-pointed curves of genus zero, Transactions of the American Mathematical Society 330 (1992), no. $2,545-574$.

[Knu83] Finn F. Knudsen, The projectivity of the moduli space of stable curves. II. The stacks $M_{g, n}$, Mathematica Scandinavica 52 (1983), no. 2, 161-199.

[KV07] Joachim Kock and Israel Vainsencher, An invitation to quantum cohomology, Progress in Mathematics, vol. 249, Birkhäuser Boston, Inc., Boston, MA, 2007, Kontsevich's formula for rational plane curves.

[Lam70] Gerard Laman, On graphs and rigidity of plane skeletal structures, Journal of Engineering Mathematics 4 (1970), 331-340.

[Mum65] David Mumford, Geometric invariant theory, Ergebnisse der Mathematik und ihrer Grenzgebiete, Neue Folge, Band 34, Springer-Verlag, Berlin-New York, 1965.

[Mum77] _ Stability of projective varieties, L'Enseignement Mathématique. Revue Internationale. IIe Série 23 (1977), no. 1-2, 39-110.

[NP19] Ilia I. Nekrasov and Gaiane Yu. Panina, Compactifications of $M_{0, n}$ Associated with Alexander Self-Dual Complexes: Chow Rings, $\psi$-Classes and Intersection Numbers, Proc. Steklov Inst. Math. 305 (2019), 232-250.

[NSTW18] Anthony Nixon, Bernd Schulze, Shin-ichi Tanigawa, and Walter Whiteley, Rigidity of Frameworks on Expanding Spheres, SIAM Journal on Discrete Mathematics 32 (2018), no. 4, 2591-2611.

[Pog73] Aleksei V. Pogorelov, Extrinsic geometry of convex surfaces, American Mathematical Society, Providence, R.I., 1973, Translated from the Russian by Israel Program for Scientific Translations, Translations of Mathematical Monographs, Vol. 35.

[Pol27] Hilda Pollaczek-Geiringer, Über die Gliederung ebener Fachwerke, Zeitschrift für Angewandte Mathematik und Mechanik (ZAMM) 7 (1927), 58-72. 
[Sha13] Igor R. Shafarevich, Basic algebraic geometry 1. Varieties in projective space, third ed., Springer, Heidelberg, 2013.

[ST10] Reinhard Steffens and Thorsten Theobald, Mixed volume techniques for embeddings of Laman graphs, Computational Geometry 43 (2010), no. 2, 84 - 93, Special Issue on the 24th European Workshop on Computational Geometry (EuroCG'08).

[SW07] Franco V. Saliola and Walter Whiteley, Some notes on the equivalence of firstorder rigidity in various geometries, Available at arXiv:0709.3354. 